\title{
Visión de la Salud en Apocalipsis
}

\author{
Vision of Health in Revelation
}

\begin{abstract}
Resumen
En este artículo se propone buscar en algunos textos del Apocalipsis elementos que ayuden a la comprensión de la salud como un bien que debe estar unido a la habitación en un ecosistema sano. Se muestra que la medicina ha sido entendida como un factor de normalización para sostener ecosistemas capitalistas que llamaremos bestiales. Salud y ecología son dos disciplinas que de concebirse separadas pueden ayudar a sostener ecosistemas opresores, enfermantes.
\end{abstract}

Palabras claves: Salud - Ecología - Apocalipsis - Capitalismo

\begin{abstract}
In this paper we are going to look for some texts of Revelation which help to recognise that the health is a good that should be united to a life in a healthy ecosystem. The medicine system has been working as a normalization factor for sustain capitalist ecosystems which we will call bestial. Health and ecology are two disciplines that if we understand them in isolated way it will support oppressive and unhealthy ecosystems.
\end{abstract}

Key words: Health - Ecology - Revelation - Capitalism

\section{Para ir comenzando...}

Hoy en día vivimos una época en que las ideas de salud y normalidad se han equiparado notablemente. No digo que sea una asociación nueva, claro, ya que la podemos leer desde el Antiguo Testamento en muchas de las normas de pureza e impureza como también en los análisis realizados en su momento por Foucault. Destaco en este momento el libro "Lo normal y lo patológico" de Georges Canguilhem (Canguilhem, 1971), pensando esta relación que luego es releída por Foucault dando un giro fuertemente social, político y cultural.

Sin embargo, hoy en día el desarrollo de la medicina ligada al sistema capitalista de mercado ha conllevado una medicalización de la vida humana tan grande como también más alejada del común de la gente. Así, 
una vez más, la salud y la belleza, la salud y la fortaleza y en definitiva la salud y la normalidad se erigen como marcos desde los cuales en nuestra sociedad nos relacionamos, percibimos o buscamos vivir.

En este sentido, pongo un par de ejemplos. La misma medicina es la que nos ha sugerido que el cuerpo de la mujer es más débil que el del hombre y por eso necesita de un control, cuidado y asistencia permanente mientras que los cuerpos de varones pareciera que no necesitan de la medicina tanto como el de las mujeres. La medicalización ha sido otra de las herramientas en este construir nuestro mundo patriarcal. Otro claro ejemplo es la medicalización de la infancia en busca de controlar actitudes que no se enmarcan en la "normalidad" escolar. Así tenemos hoy en día la creciente tipificación de síntomas para los cuales se corresponde una medicina. Y el mercado, en esto, sigue siendo pionero...Para el caso de la medicalización cito un artículo del periódico argentino Página 12:

En nombre de la salud y la normalidad observamos un empuje que lleva a la ciencia hacia el dispositivo del discurso capitalista y produce el plan macabro de una sociedad medicalizada a través de la expansión de diagnósticos en serie y a medida de las necesidades de los grandes laboratorios. Uno de los mayores éxitos del neoliberalismo es haber instalado dos creencias generalizadas que responden sugestivamente a la alianza entre neurociencias e industrias farmacológicas: la de una supuesta normalidad psíquica que se debe alcanzar y que la vía para esa consecución es la medicalización. (Merlin, 2019)

Pero esta preocupación de la medicina como una ciencia que "normaliza" tiene que ser puesta en relación con otra ciencia de nuestros tiempos: la ecología. Y esta relación tal vez sea más cercana a nuestros tiempos, por lo menos en occidente. Pero lo cierto es que no hay una construcción popular que relacione la medicina con la ecología. Parecieran dos preocupaciones diferentes, dos ciencias que no tienen por qué vincularse. Al menos, una vez más, en occidente.

Creo que esta des-vinculación entre lo que se refiere a la salud y lo que se refiere a nuestros medios ambientes es una herramienta fundamental en el funcionamiento de este sistema social, económico y cultural que está destruyendo el planeta y ciertamente a los seres humanos.

En este momento es central volver a pensar la salud y la ecología como esferas mutuamente relacionadas.

La pregunta entonces es si en un texto como el Apocalipsis de Juan podremos encontrar algo que nos ayude a pensar desde aquellos textos nuestra vida de hoy.

Nuestra presuposición es doblemente afirmativa. En primer lugar, porque la apocalíptica, al resistir contra invasiones político-económico-culturales, tuvo que replantearse los criterios de normalidad que se iban imponiendo desde el poder. Entonces sospechamos que de por sí el texto 
apocalíptico conlleva una fuerte crítica hacia lo que se propone como normal o anormal. Y esto lo veremos específicamente en la idea de salud que se puede presentar en un texto como Apocalipsis.

En segundo lugar, creemos que la apocalíptica trae consigo la idea propia del mundo semítico en donde el shalom era un buen vivir, sanidad, una buena tierra cuidada. La tierra, el agua, el aire eran parte del mundo en donde debían habitar seres humanos sanos.

Veamos entonces un poco el texto apocalíptico

Busquemos términos que estén en la esfera semántica de la salud. Notamos dos conceptos que tienen que ver con esto. En primer lugar, el verbo therapeuo, curar. En segundo lugar, muy relacionado con éste, el sustantivo therapeia. Finalmente, desde el término farmakeia/farmakos nos adentraremos en la visión de sanidad en Apocalipsis.

\section{Therapeuo, la salud bestial}

Así es que nos focalizamos entonces ahora en el verbo "curar", therapeuo, en el capítulo 13,3 referido a la herida mortal de la Bestia:

Ví una de sus cabezas como herida de muerte, pero su herida mortal se había sanado (etherapeuthe). Y toda la tierra se maravilló (ethaumasthe) en pos de la bestia. Ap. 13,3

Esta herida, nos va a decir el texto, es curada y este evento de curación produce en quienes lo observan un fenómeno: el seguimiento del ser curado: la Bestia. El mismo verbo se encontrará también referido al mismo tema en 13,12:

Y ejerce toda la autoridad de la primera bestia en presencia de ella, y hace que a tierra y sus habitantes adoren a la primera bestia cuya herida mortal fue sanada (etherapeuthe). Ap 13,12

Es interesante poder remarcar que lo que es curado en este capítulo 13 es la herida de la Bestia. Podemos decir que la bestialidad misma es curada. Esta curación es la que provoca un seguimiento, un asombro de parte del grupo nombrado "habitantes de la tierra". En el versículo 12 incluso al recordar este evento de curación se mencionará la adoración de la Bestia.

A primera vista me gustaría señalar esta idea de la curación del poder como un elemento que es trabajado y utilizado por el poder mismo para generar deslumbramiento, seguimiento y adoración.

Aquí llamaría a la curación una bestialidad. La curación es lo que permite que siga adelante la opresión. No es curación del pueblo, no es curación de las injusticias que provocan enfermedades en las poblaciones. Es curación del origen mismo del dolor, es curación del centro desde el 
cual surge la muerte. Esta paradoja no debe dejar de preocuparnos. Lo sano es lo bestial en este relato. De hecho, en el final del capítulo podemos observar la posibilidad de muerte para quienes no acepten a esa Bestia curada.

Y en este final del capítulo 13 parece que la sanidad tiene un fuerte correlato con la pertenencia a un sistema financiero-económico. No se puede comprar ni vender si no se tiene la marca de la Bestia.

Y ella hace que a todos, a pequeños y a grandes, a ricos y a pobres, a libres y a esclavos, se les ponga una marca en la mano derecha o en la frente, y que nadie pueda comprar ni vender, sino el que tenga la marca, es decir, el nombre de la bestia o el número de su nombre. Ap. 13,16-17

Vemos acá cómo se unifican las dos esferas que habíamos mencionado en un primer momento y en nuestros tiempos aparecen como desvinculadas. La esfera de la salud hace normales a quienes poseen una marca corporal. Justamente cuando hablamos de medicina pensando en el cuerpo es que el texto del capítulo 13 nos muestra seres con marcas corporales. Estas marcas corporales en frentes, manos, son el producto del tratamiento de la Bestia, son las marcas de la normalidad o, deberíamos decir, las marcas de la sanidad bestial.

Pero esas marcas que son propuestas como "lo normal" son las que permiten la inserción de estos seres curados, los habitantes de la tierra, en un ecosistema. Este ecosistema es lo económico, este sistema es la pertenencia a poderes bestiales. La salud de la Bestia, promulgada por la segunda Bestia es el origen de un ecosistema que se caracteriza por el intercambio comercial: comprar y vender.

Esto es creación de un ecosistema que tenderá a verse como normal, como el único posible de habitar. Es un ecosistema construido a partir de la Bestialidad, pero sobre todo es un ecosistema construido. Socialmente construido. Y es tan fuerte la idea de normalidad que ese sistema propone que, si una persona no se adapta a vivir en él, muere. $\mathrm{O}$, mejor dicho, es matada.

Entonces, la salud es lo que establece una normalidad. La normalidad es lo Bestial. Y esa salud produce un ser humano que habita un ecosistema creado por la Bestialidad. El sistema de intercambios económicos que producen una idea de normalidad al matar a quienes no se adaptan a éste.

Hasta aquí me gustaría hablar de la bestialidad como una forma de normalidad. Y de la medicina como soportando, ayudando a esta bestialidad. Remarco acá nuevamente lo dicho anteriormente con relación a la fuerte medicalización de los niños y niñas cuando no cuadran en lo que se entiende como "normal" en las instituciones escolares. Cantidades de nuevas denominaciones de síntomas y a cada uno de los cuales corresponde una medicina. Mientras tanto la bestialidad de la sociedad sigue 
andando, curada, sana. En definitiva, lo que se medica en los niños y niñas es la no adaptación a sistemas donde tienen que aprender a producir. No pueden estar sin producir o bien no pueden ser impedimentos para la producción de otros y otras.

De igual modo no puedo dejar de leer este texto desde nuestros contextos latinoamericanos recordando por ejemplo la crisis del 2001 en Argentina cuando las entidades bancarias retuvieron los ahorros de la población para no quebrar ellas mismas. Fue una de las estafas financieras más grande que se vieron en mi tiempo (hoy al momento de escribir esto tengo que decir que han sucedido nuevas estafas, más sutiles, más dañinas). Pero la razón era muy parecida a la que leemos en Apocalipsis 13: curar la Bestia sobre todas las cosas, sanar el sistema financiero para que pueda seguir funcionando aún a costa de seguir enfermando a cada habitante del país. Dejo a cada lector o lectora la tarea de hacer la propia equiparación con la situación social que vive en las diferentes regiones latinas. Seguramente sobran los ejemplos de curación de la bestia.

\section{La otra sanidad, therapeia}

En el capítulo 22 volvemos a encontrarnos ahora con el sustantivo Therapeia. Me parece sumamente interesante que Apocalipsis utilice la misma raíz semántica que el capítulo 13. Esta inclusión nos pide comparar, oponer, o por lo menos tratar de relacionar aquella curación vista en el capítulo 13 con esta que aparece en el capítulo 22.

Después me mostró un río de agua de vida, resplandeciente como cristal, que fluye del trono de Dios y del Cordero. En medio de la avenida (plateias) de la ciudad, y a uno y otro lado del río, está el árbol de la vida, que produce doce frutos, dando cada mes su fruto. Las hojas del árbol son para la sanidad (therapeian) de las naciones (ethnwv). Ap. 22,1-2

El término lo vemos específicamente en 22,2 aunque está en un contexto narrativo que me interesa particularmente leer con atención. En este texto se puede reconocer, entre otras cosas, la estrecha relación entre la salud y la ecología. Algunas pistas para considerar:

1. Lo primero que aparece es un río. Es un río de agua de vida, resplandeciente. No puedo dejar de pensar en nuestros ríos en Latinoamérica. Ya no son resplandecientes, ya no son aguas de vida en su mayoría. Hemos aprendido (mal-aprendido claro) en nuestras sociedades industriales que el río es el vertedero de nuestras basuras, de nuestros desperdicios. Así hemos llegado a contaminar profundamente los ríos. Ríos que difícilmente puedan llevar vida sino más bien basura y la más de las veces tóxicos industriales o agrícolas. El agua como elemento de salud, de bienestar ahora la tenemos que comprar en botellas o comprar 
filtros. El agua como elemento de curación ya no está hoy disponible gratuita ni saludablemente.

2. Lo segundo es que la ubicación de ese río es en medio de la plateia, de la plaza de la ciudad. Si algún río, ocasionalmente, quedara limpio seguramente no sería en una de nuestras ciudades. Nos hemos acostumbrado a la prohibición de bañarnos en los ríos de nuestras grandes ciudades. Este río cristalino que atraviesa la ciudad es denominado río de aguas de vida. ¡La ciudad regada por la vida! ¿Podremos pensar en nuestras megalópolis como regadas por vida? ¿Cómo armar ríos de vida en nuestras civilizaciones violentas?

3. Lo tercero es que, a la orilla del río, o ambas orillas, crece este árbol de vida. Sin dudas recordando aquel del paraíso. Tal tiempo primigenio a veces nos lleva a olvidar la fuerza simbólica del río en medio de la ciudad y de su alimento al árbol, también en medio de la ciudad. La ciudad que se erige como símbolo de civilización en tantas culturas ha eliminado la naturaleza de su medio. Aquí la cultura romana es un vivo ejemplo de la civilización urbana por excelencia. Este texto no deja de ser una denuncia en este sentido.

4. Lo cuarto es que el árbol produce frutos. El fruto en tanto alimento no está en un negocio no está en manos privadas sino en medio de la plaza. No es un fruto/alimento que se agote, sino que el texto menciona que va dando fruto/alimento a lo largo del año. La salud y la alimentación es un tema que nos provoca constantes luchas, constantes denuncias. La alimentación es vista aquí como parte del sistema de salud, del sistema de vida. Agua, alimento y salud. Comparto un relato del pueblo wichí en donde se puede notar este cambio en la relación con el alimento y su adquisición. Notemos cómo la disponibilidad del alimento tiene que ver con todo un ecosistema que cambia:

"Antes de que llegara la gente criolla y los comerciantes, los wichí comían todas las cosas, la comida natural. El monte era rico, virgen, abundaba toda clase de comida de distintos gustos. Ellos no conocían la mercadería de los almacenes, por eso tenían dientes sanos y pocas enfermedades, porque no había otra gente, no había contagio.

Los wichí cazaban los bichos para su consumo, no más de lo que necesitaban, ellos cuidaban mucho el monte y los bichos, no cortaban los árboles, por ejemplo si hay miel arriba de un árbol, no lo corta; ellos nada más tenían las sogas o cuerdas especiales para subir arriba de un árbol y sacar la miel.

Después vinieron los criollos y otros ya empezaron a explotar el monte cortando la madera. Los comerciantes compraban cueros 
de los animales, entonces el wichí ya empezaba a matar los bichos para vender los cueros a los comerciantes y así canjear un poco de mercadería" (Avendaño, 2016 pp. 60-61)

5. Lo quinto es que el árbol produce hojas que sirven para curación de las ethnes. Aquí podemos tener en cuenta la múltiple significación del término ethnes. Podemos recordar que puede significar pueblo, gente, nación, gentiles. Cualquiera de estas acepciones me lleva a pensar, pero me quedo aquí en dos de ellas. Por un lado, estamos ante una medicina que llega al pueblo, no es mediada por ninguna industria. El acceso a la salud es público, no hay restricciones sino más bien apertura, donación. Por otro lado, es una medicina que llega a gentiles entendiendo a gente que no habitaba en la ciudad. Aquí podemos tener en cuenta otro de los temas cruciales en nuestros tiempos salud y migrantes. $Y$ no quiero dejar de notar que la medicina no es para los originarios de la ciudad solamente sino para las gentes que puedan no ser de la misma.

6. Del versículo 3 al 5 podemos leer algo así como el resultado de este ecosistema nuevo: agua pura, alimento y medicina. El encuentro de una vida plena. Como donación para todos y todas.

A modo de resumen en este segundo uso del concepto de salud, podemos afirmar que hay una notable contraposición con el uso bestial. Podemos entonces denunciar dos usos contrarios del concepto de salud. Por un lado, la salud bestial que cura al poderoso y su ecosistema, para seguir explotando. Por otro lado, en oposición, la salud de vida que hidrata, alimenta y cura a todas las naciones, todo el tiempo.

Si uno pudiera ver los resultados de ambas medicinas encontraríamos que la medicina bestial tiene como resultado lo que conocemos como el 666. Aquí me detengo a pensarlo como el ecosistema económico bestial en el que todos y todas somos inyectados. De hecho, la muerte se apodera de aquellos y aquellas que no aceptan la marca de la Bestia, la marca que anuncia su "curación" bestial. El resultado final lo podemos encontrar en la descripción del capítulo 17 y 18 donde se nos muestra la voracidad de la Gran Ciudad. La voracidad que se erige a partir de comerciantes, capitanes de barco, reyes. Los "sanos bestiales" que van consumiendo los recursos de la Tierra. La acumulación de capital como dato de sanidad bestial. La acumulación y la destrucción del ambiente en cada uno de los productos que se detallan.

Muy por el contrario, el resultado de la medicina que encontramos en el capítulo 22 tiene como destinatarios a los que necesitan de agua, alimento y curación. Tiene como resultado aguas de vida. Y subrayo lo dicho anteriormente: una medicina al alcance de migrantes, del pueblo en general. Una medicina que tiene que ver también con el alimento y el agua. 
Quiero señalar que en esta idea de salud que vemos en el capítulo 22 no puedo notar el dato de acumulación que se ve en la anterior. La simple$\mathrm{za}$, inclusive el pasar del río, como un elemento necesario para el bienestar de la salud. No hay acumulación de frutos, no hay acumulación de hojas. Simplemente están para ser llevadas.

Pero también hay otra idea que es necesario remarcar. La sanidad del capítulo 22 se da en un marco ambiental. Se vuelve a pensar en lo ecológico, en la ciudad, pero con la inserción del río, de la vegetación. La sanidad no está fuera de lo ecológico. En este sistema ecológico si hay algo que falta es el dinero, es la transacción comercial. Esto pareciera ser pasado de largo, pero en la descripción de la ciudad no hay mención de dinero. De hecho, las riquezas como piedras preciosas u oro están disponibles para todos y todas en la ciudad. Pareciera que la idea de libre disponibilidad del alimento y la salud está enmarcando este ecosistema. Ya vimos en el relato wichí anteriormente compartido como se transforma esta libre disponibilidad del alimento cuando el ecosistema cambia.

\section{La farmakeia}

Hay otro uso de la idea de salud que tenemos que revisar en su relación con la ecología. Lo encontramos en varios lugares en el Apocalipsis: 9,$21 ; 18,23 ; 21,8$ y 22,15. El término es farmakeia/farmakós.

No es éste un término propio del Apocalipsis. Lo podemos encontrar por ejemplo en el Nuevo Testamento, también en Gal 5,20. Aquí será concebido como parte de un listado de obras de la carne.

Pero ¿De dónde nos llega este término? Es notoria su apreciación negativa en el texto bíblico del Nuevo Testamento, así como también en el Antiguo. Antes de revisar su origen en diferentes tradiciones del Antiguo Testamento, me parece oportuno decir algo sobre las traducciones que se han hecho de farmakeia/farmakos. No deja de ser preocupante la traducción de este término al español. Dicha traducción ha inducido a atacar tradiciones divergentes de la medicina occidental. A modo de ejemplo: Reina Valera traduce hechiceros/hechicería mientras que Dios Habla Hoy lo hace como brujería/los que practican la brujería. Nueva Versión Internacional traduce como artes mágicas/los que practican las artes mágicas. Otras traducciones: sortilegios (Biblia La Palabra en Ap 18:23). ¿Por qué veo como algo preocupante estas traducciones? En muchas de nuestras culturas la idea de brujería o hechicería fue vinculada con las medicinas de nuestros pueblos originarios. Y no me limito sólo a las traducciones bíblicas, sino que tenemos que remontar a toda una ciencia antropológica que concibió las acciones curativas no occidentales como brujería o magia (Strauss, 1949) De más está entonces decir que el resultado de esta traducción sea muy probablemente la condena de dichas medicinas. Y consecuentemente, la naturalización de la medicina occidental como la única 
viable y posible. Y esto, esta traducción, es algo que tenemos que sanar...

Volvamos al texto bíblico. Recorremos, muy brevemente, el uso del concepto en la Septuaginta, encontrando este valor negativo de farmakoi por ejemplo en Dt 18:10 donde se prohíbe el uso de contactos con tradiciones paganas.

Pero tal vez sea más adecuado a nuestro contexto apocalíptico ubicarnos en el uso de farmakoi en el relato del Éxodo. Así tendremos Ex 7:11 donde el Faraón convoca a "sabios" (sofistas) y "hechiceros" (farmakoi) para contraponerse a las acciones milagrosas de Moisés. Dice también la LXX en Ex 7:11 que los epaoidoi (traducido también por hechiceros) hicieron encantamientos (farmakeia). Aquí vemos que estos epaoidoi quedan vinculados a su acción, farmakeia. Este uso continúa en Ex 7:21 mencionando a los epaoidoi con las farmakeia. En Ex 9:11, curiosamente, los farmakoi no pueden permanecer sanos ni curar su propio sarpullido cuando Moisés realiza una de las plagas para liberar a su pueblo.

Vemos también que Malaquias 3:5 pone a farmakoi juntamente con otros que engañan al pueblo, siguiendo muy posiblemente la tradición de Éxodo en lugar de la tradición deuteronómica.

Quisiera entonces detenerme para señalar un par de elementos. En primer lugar, que la farmakeia no podemos considerarla como una sabiduría medicinal que es descartada por ser extraña a la cultura hebrea ${ }^{1}$. Este significado me parece importante sacarlo del medio sobre todo en momentos donde se está revalorizando, y en muchos casos rescatando, la medicina de nuestros antepasados y antepasadas. Esta búsqueda en tradiciones medicinales de nuestros pueblos originarios ciertamente es fuertemente criticada desde una industria farmacéutica (¿Acaso, pienso ahora, este término español proviene de farmakeia?) No es esto, definitivamente, lo que podemos encontrar en el contexto de nuestro texto bíblico. Creo que la traducción "hechiceros" o "brujería" no ayuda en este sentido puesto que este vocablo se ha relacionado en muchas de nuestras culturas latinoamericanas con los y las personas que conocen las tradiciones medicinales antiguas, milenarias. $\mathrm{Y}$ a estas personas muchas de nuestras tradiciones cristianas las han condenado, tristemente... (Mamani, 2010 pp.14-21)

Para una discusión sobre este tema remito a un par de artículos que trabajan reflexionando sobre la medicina de pueblos originarios de los Andes con relación a la tradición cristiana (Zambrano y Huacani, 2006) En ambos artículos el tema de la traducción de farmakeia es un problema puesto que asumen la idea del término en la tradición bíblica como refiriendo a las sabidurías extrañas al pueblo hebreo. Así se produce la

\footnotetext{
${ }^{1}$ Esta idea de atacar un conocimiento extranjero sí puede verse en el texto anteriormente citado de Deuteronomio. Pero no es la tradición más significativa o que por lo menos se toma con más fuerza en las relecturas profética y apocalíptica.
} 
asociación de este vocablo con las tradiciones propias de las culturas andinas, principalmente la aymara.

En segundo lugar, señalaré que esta farmakeia y sus administradores, los farmakoi, son asociados en nuestro texto bíblico, fundamentalmente a partir del relato de Éxodo, con los que buscan evitar la liberación del pueblo. Los farmakoi son esencialmente trabajadores en favor del poder opresivo. Usan su saber para bien de los poderosos. Mientras Moisés busca liberar al pueblo con sus acciones milagrosas, estos farmakoi buscan sostener el status quo: de los esclavos y del poder gobernante.

De modo que resulta interesante remarcar que este término reaparezca en la narrativa apocalíptica como un elemento a desterrar. Si recordamos la fuerte tradición del Éxodo como trasfondo del Apocalipsis de Juan, entonces podemos cerrar mucho más este concepto y su vinculación a los que engañan con medicinas falsas a los pueblos. El tema del engaño, entonces, quiero unirlo a la therapeia que vimos en el capítulo 13 en donde el tema del engaño está presente en un modo total. Aquí Apocalipsis 18,23 nos sirve de resumen:

Tus mercaderes / comerciantes eran los grandes de la tierra, porque con tus farmakeia engañaste a todas las naciones.

Pero no por ser resumen quiere decir que no esté fuertemente unido a una importante tradición histórica de ver en la farmakeia una herramienta de engaño. Podemos ver en este caso un texto que también evidencia ser marco para la narrativa de Apocalipsis 17-18:

"Esto sucederá debido a la multitud de las fornicaciones de la prostituta, de bella apariencia y experta en hechizos (farmakon), que seduce a las naciones con sus fornicaciones y a los pueblos con sus hechizos (farmakois)" Nahum 3,4

Tal vez, a modo de sugerencia, podemos pensar que una mejor traducción para farmakoi sería "encantadores" en cuanto producen visiones que alejan de la liberación. Igualmente sería mejor traducir y comprender la farmakeia como encantamientos.

En el nuevo mundo, ese nuevo ecosistema propuesto en Apocalipsis, estos encantadores son desterrados (Ap.22,15) puesto que pertenecen a la lógica del viejo ecosistema.

\section{Salud, ecología, bestialidad, encantamientos, encantadores}

¿Cómo juntar entonces todo esto visto hasta aquí para encontrar en el texto de Apocalipsis un mensaje para nuestros tiempos? Veamos algunas conclusiones que encuentro a partir de estas lecturas apocalípticas. 


\section{a. Apocalipsis habla de ecología en relación con la salud hu- mana}

Pongo esto en relación puesto que en la actualidad no lo veo demasiado unido. Todo lo que se entiende en nuestros tiempos con lo ecológico no está, al menos a nivel popular y masivo claro, vinculado con el ser humano enfermo, con la persona doliente. Es de vital importancia vincular la acción de protección ecológica con el bienestar humano. Aunque hoy en día esto está poniéndose sobre la mesa no pareciera que la acción farmacéutica sea modificada por la acción ecológica y viceversa.

\section{b. Apocalipsis habla de salud humana en relación con la eco- logía}

Quiero subrayar fuertemente este punto. No puede haber salud en un medio ambiente enfermo. No puede ser curado o curada quien está habitando una bestialidad. Toda la farmacéutica occidental fracasará si sigue siendo pensada como algo que ataca un problema personal y no revisa la prevención, el bienestar de la persona que habita un determinado ecosistema. Y como vimos anteriormente, un ecosistema que la más de las veces es creado a partir de una bestialidad.

\section{c. Apocalipsis revela que hay formas sanas de curar y formas enfermas de curar}

Hemos elevado a diosa a la medicina occidental. Y lo cierto es que en muchos casos es de gran ayuda. Pero también es cierto que esta medicina rompe la relación persona-mundo. No la considera en su acción sanadora. Es una ciencia sumamente fragmentaria y fragmentante.

En este sentido, poder aprender de otras ciencias medicinales y sus concepciones holísticas nos ayudará a recomponer la humanidad en su mundo. Si bien no estamos descartando los inmensos avances de la medicina occidental, es de suma urgencia que esos avances sean puestos en un marco ecológico. De otro modo sólo se estará curando la herida de la Bestia.

\section{d. Apocalipsis revela que lo ecológico está atado a la cultura, al engaño producido en ella}

¿Podremos tener éxito en la lucha ecológica? ¿Nos dirigimos a un precipicio como humanidad? ¿Nos estamos suicidando colectivamente? Tal vez el Apocalipsis alerta sobre esto que es esencial: la cultura en la que vivimos, esa cultura devoradora, esa cultura que construye seres humanos que consumen por demás, muy por demás (claro que esto se da mientras otros y otras en mayor número mueren de hambre, sed, etc) es la principal causante de la muerte lenta de nuestro mundo. $Y$ de nuestra especie. 
La farmakeia hoy es disociar lo que en los puntos anteriores hemos tratado de asociar: la cultura, el modo de vivir en el mundo, el modo de pensarnos como seres humanos, el modo de asociarnos con el mundo, con el otro/la otra.

La farmakeia hoy es todo un sistema perverso que nos impulsa a luchar por "lo ecológico" mientras seguimos siendo parte de una cadena devoradora de humanos, de naturaleza, de vida.

En este sentido tendremos que tener mucho cuidado con el llamado "capitalismo verde". O, para darle un término que suene más cool, "green capitalism". La idea de que el mismo capitalismo puede generar acciones que promuevan el cuidado de la naturaleza es una idea que tenemos que denunciar por ser mentirosa en sí misma. Hoy es una de las farmakeia más grande. Es la ilusión que nos presenta un sistema bestialmente devorador de que de él mismo puede surgir la solución.

El "capitalismo verde" no busca una nueva relación con el mundo y quienes en él habitan. El "capitalismo verde" busca nuevas formas de inversión, nuevas formas de producción de capital al costo que sea (Sánchez, 2018) El "capitalismo verde" sigue creando un mundo: el bestial, el que consume en forma devoradora

Y es para denunciar. Aquí vemos también la fascinación de los habitantes de la tierra. Esos habitantes que creen (sí, esta es la palabra, puesto que es una cuestión de fe) que el capitalismo puede generar la cura. Y nos encontramos por ejemplo con la idea de que la tecnología es la herramienta para esto. La tecnología puesta al servicio de un mundo mejor. Ciertamente suena sumamente interesante, atractivo. Pero es la Bestia con voz de Cordero. La tecnología no es más que una creación humana dentro de un ecosistema capitalista, ¿Por qué habría de volverse en contra de ese sistema? En este sentido es necesario denunciar las postverdades o para ponerles otro nombre, las mentiras o farmakeias. Esto es parte del movimiento ecológico-político que busca remarcar la vinculación del poder con la preocupación ecológica (Neimark, 2019).

Algo similar notamos que sucedió con el surgimiento de internet. Se comenzó a pensar en una democratización, en una cultura hippie, o punk, promovida por un libre acceso a la información y la distribución de bienes. Pero ¿Fue así? ¿O simplemente creó la ilusión de esto? Las farmakeia del sistema ecológico capitalista...

\section{e. Apocalipsis propone un modo de ser sano, sana en el mun- do nuevo}

Revisar nuestra propuesta de sanidad hoy es central. Es central porque la sanidad no puede ser otra que luchar por el agua, por el alimento, por la posibilidad de therapeia para todos y todas siempre.

FFFF 
Cualquier otra lucha denominada ecológica puede caer en ser algo más del sistema de farmakeia, de encantamientos. De esos encantamientos que nos hacen creer que luchamos por el bien, nos liberan de una supuesta culpa. Como hemos dicho anteriormente.

Pero sobre todo Apocalipsis nos recuerda que se puede vivir en mundos diferentes. Lo primero es captar que no es posible la convivencia del mundo Bestial con el mundo nuevo. Uno es el mundo con una ecología capitalista, bestial. Otro es el nuevo mundo con cielos nuevos y tierras nuevas. $\mathrm{Y}$ en esto Apocalipsis es determinante. No hay convivencia. No puede haberla. $\mathrm{Y}$ esto es marcado de diferentes formas. Lo que hemos tratado de mostrar es que el mundo nuevo cura de un modo radicalmente opuesto al mundo bestial, capitalista, consumidor. Y esta forma de concebir la sanación es una pista que podemos seguir para reconocer qué mundo habitamos.

\section{Referencias bibliográficas}

Avendaño, Ernesto; Relatos Wichí. Recopilación; Resistencia Chaco; ConTexto; 2016

Canguilhem, Georges; Buenos Aires; Siglo XXI Argentina Editores; 1971. Primera edición en francés en 1966

Huacani, Cocaure, Víctor; Sanación en el mundo aymara y en la Biblia. Pp. 363-365; en Teología andina. El tejido diverso de la fe indígena. Tomo II. ISEAT; La Paz; Editorial Plural; 2006.

Mamani Bernabe, Vicenta; El Apthapi teológico desde las mujeres aymaras. En RIBLA, 65 2010/1; pp. 14-21. Quito.

Merlin, Nora; El feliz mundo neoliberal. Nota del periódico Página 12. 1 de junio de 2017. https://www.pagina12.com.ar/41342-el-feliz-mundo-neoliberal

Consultado el 5 de marzo de 2019

Neimark, B et als; (2019) Speaking Power to "Post-Truth": Critical Political Ecology and the New Authoritarianism; en Annals of the American Association of Geographers. https: / / doi.org/10.1080/24694452.20 18.1547567 consultado el 24 de febrero de 2019

Sánchez de Jaegher, Carolina; Saving the Planet with Green Capitalism? Or re-signifying our existence with Mother Earth? The Mapuche-Valdivian case against the wind farm Pililín in the South of Chile.; en Forum for inter-American Research Vol 11.3 (Dec 2018) pp. 32-46

Strauss, Levi; El Hechicero y su magia; en Les Temps Modernes, Año 4, N 41; 1949; pp. 3-24

Zambrano, Luis; El problema del mal en el mundo andino. pp. 312-314, en

Teología andina. El tejido diverso de la fe indígena. Tomo II. ISEAT; La Paz; Editorial Plural; 2006. 